TRANSACTIONS OF THE

AMERICAN MATHEMATICAL SOCIETY

Volume 348, Number 12, December 1996, Pages 4919-4938

S 0002-9947(96)01799-0

\title{
ERGODIC PROPERTIES OF REAL COCYCLES AND PSEUDO-HOMOGENEOUS BANACH SPACES
}

\author{
M. LEMAŃCZYK, F. PARREAU, AND D. VOLNÝ
}

\begin{abstract}
Given an irrational rotation, in the space of real bounded variation functions it is proved that there are ergodic cocycles whose small perturbations remain ergodic; in fact, the set of ergodic cocycles has nonempty dense interior.

Given a pseudo-homogeneous Banach space and an irrational rotation, we study the set of elements satisfying the mean ergodic theorem. Once such a space is not homogeneous, we prove it is not reflexive and not separable. In "natural" cases, up to $L^{1}$-cohomology, the only elements satisfying the mean ergodic theorem are those from the closure of trigonometric polynomials.

For pseudo-homogeneous spaces admitting a Koksma's inequality ergodicity of the corresponding cylinder flows can be deduced from spectral properties of some circle extensions. In particular this is the case of Lebesgue spectrum (in the orthocomplement of the space of eigenfunctions) for the circle extension.
\end{abstract}

\section{INTRODUCTION}

By $\mathbf{T}$ we will mean the circle group $\{z \in \mathbf{C}:|z|=1\}$ which most of the time will be treated as $[0,1)$ with addition $\bmod 1 ; \lambda$ will denote Lebesgue measure. Each $f: \mathbf{T} \longrightarrow \mathbf{R}$ (naturally identified with $f: \mathbf{R} \longrightarrow \mathbf{R}$ periodic of period 1), $f \in L^{1}(\mathbf{T})$ has its Fourier expansion

$$
f \sim \sum_{n=-\infty}^{\infty} \widehat{f}(n) e^{2 \pi i n x}
$$

We will only consider functions with zero mean.

For $f \in L^{1}(\mathbf{T})$ and $t \in \mathbf{T}$, we denote $f_{t}(x)=f(t-x)$. By a pseudo-homogeneous Banach space on $\mathbf{T}$ we mean a linear subspace $B$ of $L^{1}(\mathbf{T})$ with a norm $\|\cdot\|_{B}$ stronger than the $L^{1}$-norm, making $B$ a Banach space and satisfying:

(i) if $f \in B$ and $t \in \mathbf{T}$, then $f_{t} \in B$ and $\left\|f_{t}\right\|_{B}=\|f\|_{B}$. If in addition

(ii) $f_{t_{n}} \longrightarrow f_{t}$ in $B$ whenever $t_{n} \longrightarrow t$ in $\mathbf{T}$, then the pseudo-homogeneous Banach space $B$ is called a homogeneous Banach space on T. Main properties of pseudo-homogeneous Banach spaces are either contained or can be easily deduced from [9], chapter 1 ; in particular, there exists a biggest homogeneous Banach subspace $B_{h}$ contained in $B$; it is defined as

$$
B_{h}=\left\{f \in B: t \mapsto f_{t} \text { is a continuous map from } \mathbf{T} \text { to } B\right\},
$$

Received by the editors July 3, 1995.

1991 Mathematics Subject Classification. Primary 28D05, 47A10.

Research of the first author was partially supported by KBN grant 2 P301 03107 (1994).

Research of the third author was supported by grant GAUK 368 of Charles University. 
or as the closure of trigonometric polynomials in $B$.

We prove that if $B$ is not homogeneous, it is not reflexive and not separable. Moreover, we find necessary and sufficient conditions to have

$$
L^{1} * B \subset B \text {. }
$$

In particular, this is the case if the closed unit ball of $B$ is closed in $L^{1}$. This latter condition is satisfied by many "natural" examples of pseudo-homogeneous spaces.

Suppose now that $B$ is a Banach space and $T$ is an isometry on it. Assume also that zero is the only fixed point of $T$. We say that for an $x \in B$ the ergodic theorem holds true if

$$
\lim _{n \longrightarrow \infty} \frac{1}{n} \sum_{j=0}^{n-1} T^{j} x=0
$$

The set of all elements of $B$ for which the ergodic theorem holds is denoted by $\operatorname{ET}(B, T)$. An element $x \in B$ is said to be a coboundary if $x=y-T y$ for certain $y \in B$. Later we will consider the situation, where $x \in B, x=y-T y$ for $y$ belonging to some larger space; in this case we still call $x$ a coboundary; $y$ will be called a transfer function. The following theorem, due to J. von Neumann, is classical:

Theorem 1. An element $x \in B$ belongs to $\operatorname{ET}(B, T)$ if and only if $x$ is in the closure of the subspace of coboundaries (with transfer function in $B$ ).

Recall that $\operatorname{ET}(B, T)=B$ whenever $B$ is reflexive.

Assume that $\alpha \in[0,1)$ is irrational with continued fraction expansion

$$
\alpha=\left[0 ; a_{1}, a_{2}, \ldots\right]
$$

and $\left(p_{n} / q_{n}\right)$ the sequence of convergents; $q_{n}$ is said to be the $n$-th denominator of $\alpha$. Denote by $T x=x+\alpha$ the corresponding irrational translation on $X=[0,1)$. We show that if in a pseudo-homogeneous space $B,(1)$ is satisfied, then each $f \in \operatorname{ET}(B, T)$ is cohomologous to a $g \in B_{h}$ via a transfer function from $L^{1}$. In the general case, however, $B_{h}$ can be reduced to zero (as the example of singular functions with bounded variation shows) and then this property of $\operatorname{ET}(B, T)$ is no longer true. In fact, we show that if each function of $\operatorname{ET}(B, T)$ is an $L^{1}$-coboundary, then each element of $B$ is also.

Assume that $T:(X, \mathcal{B}, \mu) \longrightarrow(X, \mathcal{B}, \mu)$ is an ergodic automorphism. Each measurable function $f: X \longrightarrow \mathbf{R}$ will be called a cocycle. Then $f^{(n)}$ is defined, for $n \in \mathbf{Z}$, by $f^{(n)}(x)=\sum_{0}^{n-1} f\left(T^{k} x\right)$ if $n \geq 0$ and $f^{(n)}(x)=-\sum_{n}^{-1} f\left(T^{k} x\right)$ if $n<0$. Let $\overline{\mathbf{R}}=\mathbf{R} \cup\{\infty\}$ be the one-point Alexandroff compactification of $\mathbf{R}$. Then $r \in \overline{\mathbf{R}}$ is said to be an extended essential value of $f$ (see [23]) if for each open neighbourhood $U(r)$ of $r$ and an arbitrary set $C \in \mathcal{B}, \mu(C)>0$, there exists an integer $n$ such that

$$
\mu\left(C \cap T^{-n} C \cap\left\{x \in X: f^{(n)}(x) \in U(r)\right\}\right)>0 .
$$

The set of extended essential values will be denoted by $\bar{E}(f)$. The set $E(f)=$ $\bar{E}(f) \cap \mathbf{R}$ is called the set of essential values of $f$ and it is a closed subgroup of $\mathbf{R}$. The skew product

$$
T_{f}:(X \times \mathbf{R}, \tilde{\mathcal{B}}, \tilde{\mu}) \longrightarrow(X \times \mathbf{R}, \tilde{\mathcal{B}}, \tilde{\mu}), \quad T_{f}(x, r)=(T x, f(x)+r)
$$

is said to be a cylinder flow. Here by $\tilde{\mu}$ we denoted the product measure of $\mu$ and infinite Lebesgue measure $\lambda$ on the line. A cylinder flow is ergodic iff $E(f)=\mathbf{R}$ 
([23]); in this case the cocycle $f$ will be called ergodic. A necessary condition for an integrable $f$ to be ergodic is $\int_{X} f d \mu=0$.

If $G$ is a locally compact, second countable Abelian group (denoted additively) and $f: X \longrightarrow G$ is a measurable map, then we can easily repeat the foregoing definitions by replacing $\mathbf{R}$ by $G$. If $G$ is compact then there exist a closed subgroup $H$ (equal to the set of essential values of $f$ ) and a measurable function $p: X \longrightarrow G$ such that for the cocycle

$$
g(x)=f(x)+p(x)-p(T x) \in H
$$

the extension $T_{g}$ is ergodic as an $H$-extension. In particular, $f$ is a coboundary if and only if $E(f)$ is reduced to zero. However, if $G$ is not compact what can happen is that $E(f)$ is $\{0\}$ but $f$ is not a coboundary. According to [23], such cocycles are said to be of type $I I I_{0}$. A cocycle $f$ is said to be regular if the quotient cocycle $f^{*}: X \longrightarrow G / E(f)$ is a coboundary. Obviously, each ergodic cocycle is regular. In the case where the only compact subgroup is $\{0\}$, a cocycle is a coboundary if and only if $\infty \notin \bar{E}(f)$ (that is, there exist a set $B$ of positive measure and a compact set $K$ in $G$ such that $f^{(n)}(x) \in K$ whenever $\left.x, T^{n} x \in B\right)$. In the case $G=\mathbf{R}$ nonregular cocycles are exactly those of type $I I I_{0}$.

In some classical cases (like $L^{p}(X, \mu), L^{\infty}(X, \mu)$ ), for each ergodic $T$ real coboundaries (with measurable transfer functions) are dense in the corresponding topologies. We suppose now that $T$ is an irrational rotation on $\mathbf{T}$; then we can find pseudohomogeneous spaces where coboundaries are not dense. This is especially the case of some spaces which admit an abstract Koksma's inequality (see Section 2.2), for example $B V(\mathbf{T})$ - the space of functions with bounded variation or spaces where the norm is determined by the speed of convergence of Fourier coefficients to zero.

Ergodicity of straight lines (i.e. of cocycles $f_{c}(x)=c x-c / 2, c \in \mathbf{R} \backslash\{0\}$ ) under irrational rotations has been proved by Pask in [19] (see also [1]). We develop a new method of proving ergodicity of real cocycles based on spectral properties of the corresponding Anzai skew products $T_{\exp (2 \pi i f)}$. For example we obtain that if $f$ has bounded variation and $T_{\exp (2 \pi i f)}$ has Lebesgue spectrum in the orthocomplement of the eigenfunctions of $T$ then $E(f)=\mathbf{R}$.

This method will allow us to prove that in the space $B V(\mathbf{T})$ we have even a stability property for the ergodicity of certain cocycles. For example if $g \in$ $B V(\mathbf{T})$ is sufficiently close to a straight line $f_{c}$, then it is also ergodic (for all irrational rotations). Moreover functions which are piecewise absolutely continuous with $\int_{0}^{1} f^{\prime}(x) d x \neq 0$ enjoy the same property. In fact the interior of the set of ergodic cocycles in $B V(\mathbf{T})$ is dense.

\section{Pseudo-homogeneous spaces on $\mathbf{T}$ and Convolutions}

We discuss here the condition (1), i.e. $L^{1} * B \subset B$, for a pseudo-homogeneous space $B$ on $\mathbf{T}$. Firstly, we state some general remarks .

If (1) holds, by Closed Graph Theorem, there exists a constant $M$ such that

$$
\|g * f\|_{B} \leq M\|g\|_{1}\|f\|_{B} \quad \text { for all } f \in B \text { and } g \in L^{1} .
$$

Then, an approximation of $g$ by trigonometric polynomials shows that $g * f$ is a $B$-limit of trigonometric polynomials, and in fact $L^{1} * B \subset B_{h}$.

Besides $B$ must contain enough trigonometric polynomials. More precisely, let $\mathcal{P}=\mathcal{P}(\mathbf{T})$ be the space of all trigonometric polynomials on $\mathbf{T}$. We denote by $\Lambda(B)$ the set of all integers $n$ for which there exists some $f \in B$ with $\widehat{f}(n) \neq 0$ and 
conversely, if $\Lambda$ is a set of integers, let $\mathcal{P}_{\Lambda}$ be the space of all $P \in \mathcal{P}$ with frequencies in $\Lambda$. Since the convolution product of any $f \in B$ by the exponential $e^{2 \pi i n x}$ yields $\widehat{f}(n) e^{2 \pi i n x}$, a necessary condition for $(1)$ is

$$
\mathcal{P}_{\Lambda(B)} \subset B .
$$

We shall denote $b(B)$ the closed unit ball in $B$ and by $\bar{A}^{1}$ the $L^{1}$-closure of any subset $A$ of $L^{1}$.

Lemma 1. If $f \in b(B)$ and $g \in b\left(L^{1}\right)$, then $g * f \in \overline{b(B)}^{1}$.

In particular, if $b(B)$ is closed in $L^{1}$, then $L^{1} * B \subset B$ and $\|g * f\|_{B} \leq\|g\|_{1}\|f\|_{B}$ for all $f \in B$ and $g \in L^{1}$.

Proof. This follows elementarily from the fact that the convolution product $g * f=$ $\int f_{t} g(t) d t$ can be approximated in the $L^{1}$-norm by finite sums $\sum f_{t_{j}} \int_{I_{j}} g(t) d t$, where $\left\{I_{j}\right\}$ is a partition of $\mathbf{T}$ into small subintervals and $t_{j} \in I_{j}$.

Note that if $B$ is homogeneous, this approximation holds in the $B$-norm and $L^{1} * B \subset B$ follows similarly, with again the constant in (2) equal to 1 . We fix in the sequel $\left(K_{n}\right)$ an approximating sequence of kernels in $L^{1}$; we mean a sequence of normalized nonnegative trigonometric polynomials such that the integral of $K_{n}$ outside any given neighbourhood of 0 converges to zero. By the same argument, if $B$ is homogeneous and $f \in B$ then $K_{n} * f \rightarrow f$ in $B$.

Lemma 2. If $B$ is a homogeneous space then the unit ball $b(B)$ is closed in $B$ with respect to the $L^{1}$-norm.

Proof. Let $f \in B$ and $f_{j} \in b(B)(j \geq 1)$, with $f_{j} \rightarrow f$ in $L^{1}$. Given $n,\left(K_{n} * f_{j}\right)$ is a sequence of trigonometric polynomials in $b(B)$, which lie in a fixed finite dimensional subspace and thus converge to $K_{n} * f$ for every norm. It follows that $K_{n} * f \in b(B)$ for every $n$ and, as $K_{n} * f$ converges to $f$ in $B$, this proves $f \in b(B)$.

Proposition 1. Let $B$ be a pseudo-homogeneous space on $\mathbf{T}$ and $M$ a positive constant. The following are equivalent.

(i) $L^{1} * B \subset B$ and $\|g * f\|_{B} \leq M\|g\|_{1}\|f\|_{B}$ for every $f \in B$ and $g \in L^{1}$.

(ii) $b(B) \subset M \cdot{\overline{b\left(B_{h}\right)}}^{1}$.

(iii) $\overline{b(B)}^{1} \cap \mathcal{P} \subset M \cdot b\left(B_{h}\right)$.

Proof. (i) implies (ii): Let $f \in b(B)$. If (i) holds, we have $K_{n} * f \in M \cdot b\left(B_{h}\right)$ for every $n$, and $K_{n} * f \rightarrow f$ in $L^{1}$.

(ii) implies (iii): It is enough to prove that if $C$ is a homogeneous space, then

$$
\overline{b(C)}^{1} \cap \mathcal{P} \subset b(C) .
$$

Any polynomial in $\overline{b(C)}^{1}$ belongs to $\mathcal{P}_{\Lambda(C)}$ and, since $C$ is closed under convolutions by functions of $L^{1}$, we have $\mathcal{P}_{\Lambda(C)} \subset C$. Hence $\overline{b(C)}^{1} \cap \mathcal{P} \subset \overline{b(C)}^{1} \cap C$. Now, by Lemma $2, \overline{b(C)}^{1} \cap C=b(C)$.

(iii) implies (i): Let $f \in b(B)$ and let $g$ be a trigonometric polynomial in $b\left(L^{1}\right)$. Then $g * f$ is a trigonometric polynomial and by Lemma 1 it lies in $\overline{b(B)}^{1}$. So, if (iii) holds, $g * f \in M \cdot b\left(B_{h}\right)$. This proves $\mathcal{P} * B \subset B_{h}$ and $\|g * f\|_{B} \leq M\|g\|_{1}\|f\|_{B}$ for every $f \in B$ and $g \in \mathcal{P}$. Now (i) follows by density of trigonometric polynomials in $L^{1}$. 
Examples. It is easy to check that the unit ball of $B$ is closed in $L^{1}$ and thus condition (1) holds when $B=L^{p}(\mathbf{T})$ with $1 \leq p \leq+\infty$, or $B=\operatorname{Lip}_{\kappa}(\mathbf{T})$, the space of Hölder continuous functions with exponent $\kappa$. It is also the case for $B V(\mathbf{T})$, the space of functions with bounded variation on $\mathbf{T}$ (which we consider as a subspace of $L^{1}$-we can restrict to right-continuous $B V$-functions).

For $B=\mathrm{O}(1 / n)$, the space of functions $f \in L^{1}$ with $\widehat{f}(n)=\mathrm{O}(1 / n)$, the property (1) follows directly from $\left(|\widehat{g * f}(n)|=|\widehat{g}(n) \widehat{f}(n)| \leq|| g\left|\|_{1}\right| \widehat{f}(n) \mid\right.$. Besides, since we restrict to functions with zero mean, the norm on $B$ can be defined by $\|f\|_{B}=\sup _{n}|n \widehat{f}(n)|=\sup _{n}\left|\int n e^{-2 \pi i n t} f(t) d t\right|$; then the unit ball is closed in the $L^{1}$-topology. The same holds for spaces defined by other conditions on the speed of convergence to zero of the Fourier coefficients.

For closed subspaces of the previous examples, we have the weaker property, shared by all homogeneous spaces (Lemma 2): the unit ball of $B$ is closed in $B$ with respect to the $L^{1}$ norm. By duality, that is the case if and only if the norm on $B$ can be defined as $\sup \left\{\int f(t) h(t) d t ; f \in A\right\}$, where $A$ is some subset of $L^{\infty}(\mathbf{T})$. Then condition (iii) of Proposition 1 reduces to $\overline{b(B)}^{1} \cap \mathcal{P} \subset B$ and the necessary condition $\mathcal{P}_{\Lambda(B)} \subset B$ is sufficient.

On the other hand, the space $B$ of singular functions with bounded variation ( $B V$-functions whose derivative vanishes a.e.) yields a counter-example for (1), since $B$ does not contain any nontrivial trigonometric polynomial. In this case $\overline{b(B)}^{1}$ is the whole closed unit ball of $B V(\mathbf{T})$, and the space $A C(\mathbf{T})=B V(\mathbf{T})_{h}$ of absolutely continuous functions is the smallest homogeneous space containing $L^{1} * B$. We have more generally:

Proposition 2. If $B$ is a pseudo-homogeneous space on $\mathbf{T}$, let $\widetilde{B}$ be the subspace of $L^{1}(\mathbf{T})$ algebraically spanned by $\overline{b(B)}^{1}$, equipped with the norm such that $\overline{b(B)}^{1}$ be its closed unit ball. Then $\widetilde{B}$ is a pseudo-homogeneous space and $(\widetilde{B})_{h}$ is the smallest homogeneous space $C$ with $L^{1} * B \subset C$.

Conversely, if $C$ is homogeneous, then $\widetilde{C}$ is the largest pseudo-homogeneous space $B$ such that $L^{1} * B \subset C$.

Proof. $\widetilde{B}$ is complete since its unit ball is closed in a larger Banach space (a decreasing sequence of closed balls in $\widetilde{B}$ whose radii go to zero is also a decreasing sequence of closed sets whose diameters go to zero in $L^{1}(\mathbf{T})$ ); since it is clear that translations act isometrically on $\widetilde{B}$, we have a pseudo-homogeneous space. Now, we can substitute any homogeneous space $C$ for $B_{h}$ in Proposition 1 without changes in the proof: namely $L^{1} * B \subset C$ if and only if there exists a constant $M$ such that $b(B) \subset M \cdot \overline{b(C)}^{1}$ or $\overline{b(B)}^{1} \cap \mathcal{P} \subset M \cdot b(C)$. This implies both statements in the proposition.

We complete this section by the following general result, which is apparently new:

Proposition 3. Let $B$ be a pseudo-homogeneous space on $\mathbf{T}$. If $B$ is either reflexive, or separable, then $B$ is homogeneous.

Proof. (1) Suppose that $B$ is reflexive. Then $b(B)$ is weakly compact and the weak topology $\sigma\left(B, B^{*}\right)$ matches the weaker Hausdorff topology $\sigma\left(L^{1}, L^{\infty}\right)$ on $b(B)$. It follows that $b(B)$ is closed in $L^{1}$ whence $L^{1} * B \subset B$; by Proposition 1 , we have $b(B) \subset{\overline{b\left(B_{h}\right)}}^{1}$ (and in fact $b(B)={\overline{b\left(B_{h}\right)}}^{1}$ ). So $b\left(B_{h}\right)$ is dense in $b(B)$ in the 
$\sigma\left(L^{1}, L^{\infty}\right)$-topology, and thus in the $\sigma\left(B, B^{*}\right)$-topology. Finally, since the strong closure of a convex set in a Banach space is equal to its weak closure, $b\left(B_{h}\right)$ is still dense in $b(B)$ in the norm topology. This proves $B_{h}=B$.

(2) In general, given $f \in B$, consider $d\left(t, t^{\prime}\right)=\left\|f_{t}-f_{t^{\prime}}\right\|_{B}$ for $t, t^{\prime} \in \mathbf{T}$. This defines a complete metric group topology on the quotient group of $\mathbf{T}$ by the subgroup of periods for $f$. This topology is stronger than the usual one and if $B$ is separable it is separable; i.e. it is a Polish group topology. Then both topologies have to be equal. It follows that the mapping $t \rightarrow f_{t}$ from $\mathbf{T}$ to $B$ is continuous.

\section{Mean ergodic theorem in pSeudo-homogeneous Banach spaCes}

Suppose now that $B$ is a pseudo-homogeneous Banach space on $\mathbf{T}$. Let $T$ denote an irrational tanslation by $\alpha$. Hence $T$ acts as an isometry on $B$.

Note that $B_{h} \subset \operatorname{ET}(B, T)$; indeed any trigonometric polynomial $P$ from $B$ may be written as $P=Q-T Q$, where $Q$ is another trigonometric polynomial still in $B_{h}$.

From this we obtain a natural question to explain how big can be the "difference" between $\operatorname{ET}(B, T)$ and $B_{h}$. In particular, is it true that if $f \in \operatorname{ET}(B, T)$ then $f=g+h-T h$ for some $g \in B_{h}$ and $h \in L^{1}(\mathbf{T})$ ? Below, we will positively answer this question provided that condition (1) is satisfied.

Let $C$ be a homogeneous Banach space. Denote by

$$
\operatorname{Cob}_{T}(B, C)=\{j \in B:(\exists h \in C) j=h-T h\} .
$$

Theorem 2. Suppose that $B$ satisfies $L^{1} * B \subset B$.

Then for each $f \in \overline{\operatorname{Cob}_{T}(B, C)}$ there exists $g \in B_{h}$ and $h \in C$ such that

$$
f=g+h-T h .
$$

Proof. Since $f \in \overline{\operatorname{Cob}_{T}(B, C)}$, we can write

$$
f=\sum_{j=1}^{\infty}\left(h_{j}-T h_{j}\right)
$$

for some $h_{j} \in C$, with $h_{j}-h_{j} T \in B$ and

$$
\sum_{j=1}^{\infty}\left\|h_{j}-T h_{j}\right\|_{B}<+\infty .
$$

Let $\left(K_{n}\right)$ be a sequence of kernels as in Section 1. For each $j=1,2, \ldots$ select $n_{j} \in \mathbf{N}$ so that

$$
\left\|h_{j}-K_{n_{j}} * h_{j}\right\|_{C}<\frac{1}{2^{j}}
$$

which is possible because $K_{n} * h_{j} \longrightarrow h_{j}$ in $C$, and let

$$
g_{j}=K_{n_{j}} * h_{j}-T\left(K_{n_{j}} * h_{j}\right), \quad h_{j}^{\prime}=h_{j}-K_{n_{j}} * h_{j}
$$

so that

$$
h_{j}-T h_{j}=g_{j}+h_{j}^{\prime}-T h_{j}^{\prime} .
$$

Since translations commute with convolutions on $L^{1}$, we have

$$
\left\|g_{j}\right\|_{B}=\left\|K_{n_{j}} *\left(h_{j}-T h_{j}\right)\right\|_{B} \leq M\left\|h_{j}-T h_{j}\right\|_{B}
$$


where $M$ is a constant such that the inequality (2) holds. It follows from (3) that the series $\sum_{j=1}^{\infty} g_{j}$ converges in $B$ towards some function $g$. We have moreover $g \in B_{h}$ because it is the limit in $B$ of a sequence of trigonometric polynomials.

On the other hand, by (4), the series $\sum_{j=1}^{\infty} h_{j}^{\prime}$ converges in $C$. Let $h$ be its sum. Since both series converge in $L^{1}$, we obtain from (5)

$$
f=\sum_{j=1}^{\infty}\left(h_{j}-h_{j} T\right)=g+h-T h .
$$

Corollary 1. If coboundaries with transfer functions in $C$ are dense in $B$ then each function $f \in B$ can be written in the form

$$
f=g+h-T h,
$$

where $g \in B_{h}, h \in C$.

Since coboundaries (with polynomial transfer functions in $B_{h}$ ) are dense in $B_{h}$, we also have a converse to Theorem 2 :

Corollary 2. Suppose moreover $B \subset C$. A function $f \in B$ belongs to $\overline{\operatorname{Cob}_{T}(B, C)}$ if and only if $f \in B_{h}+\operatorname{Cob}_{T}(B, C)$. In particular, $B_{h}+\operatorname{Cob}_{T}(B, C)$ is a closed subspace of $B$.

Corollary 3. If $f \in B$ satisfies the mean ergodic theorem in $B$ then, for any homogeneous space $C$ containing $B$,

$$
f=g+h-T h
$$

for certain $g \in B_{h}, h \in C$. In particular, $f$ is cohomologous to an element from $B_{h}$ via a transfer function from $L^{1}(\mathbf{T})$.

We shall show in Example 1 below that we cannot require that the transfer function $h$ in Corollary 3 belong to $B$. Besides, Theorem 2 and the above corollaries do not hold in general if the condition (1) is not satisfied. If we consider a pseudohomogeneous space $B$ where $B_{h}=\{0\}$, like the space of singular $B V$-functions, Theorem 2 would imply that any function in $\operatorname{ET}(B, T)$ is a coboundary and Proposition 4 below would imply that any function in $B$ is a coboundary (which is clearly false for singular $B V$-functions: the cocycle $g_{\beta}=\chi_{[0, \beta)}-\beta$ where $\beta \notin \mathbf{Z} \alpha \bmod 1$ is a classical counter-example).

Proposition 4. If every function in $\operatorname{ET}(B, T)$ is a coboundary via a transfer function in $L^{1}(\mathbf{T})$, then the same holds for every function in $B$ with zero mean.

Proof. Under the hypothesis for every $f \in \operatorname{ET}(B, T)$ there is a unique $h=h_{f} \in L^{1}$ with zero mean such that $f=h-T h$. By the Closed Graph Theorem, the so-defined linear mapping from $\operatorname{ET}(B, T)$ to $L^{1}$ is continuous and there exists a constant $M$ with $\left\|h_{f}\right\|_{1} \leq M\|f\|_{B}$.

Now, let $f$ be a function in $B$ with zero mean. For $n \geq 1$ denote $f^{(n)}=\sum_{j=0}^{n-1} T^{j} f$ and let $h_{n}=(1 / n) \sum_{j=0}^{n-1} f^{(j)}$ and

$$
f_{n}=h_{n}-T h_{n}=f-\frac{1}{n} f^{(n)} .
$$

$\left(f_{n}\right)$ is a sequence in $\operatorname{Cob}_{T}(B, B)$, hence in $\operatorname{ET}(B, T)$, which is is bounded in the $B$ norm: $\left\|f_{n}\right\|_{B} \leq 2\|f\|_{B}$ for every $n$. Since $h_{n}$ has zero mean, $\left\|h_{n}\right\|_{1} \leq M\left\|f_{n}\right\|_{B} \leq$ 
$2 M\|f\|_{B}$ for every $n$ and since the mean ergodic theorem holds in $L^{1},(1 / n) f^{(n)}$ goes to 0 in $L^{1}$, so $h_{n}-T h_{n}$ converges to $f$ in $L^{1}$. It follows that $f$ itself is a coboundary $([15])$.

\subsection{Examples.}

Example 1. We will now describe the subspace $\operatorname{ET}(B, T)$ when $B$ is the pseudohomogeneous space $\mathrm{O}(1 / n)$. Namely $f$ satisfies the mean ergodic theorem in $B$ if and only if

$$
n \widehat{f}(n) \longrightarrow 0 \quad \text { whenever } n \alpha \longrightarrow 0(\bmod 1) .
$$

Indeed, let $\|x\|$ denote the distance of $x \in \mathbf{R}$ to the nearest integer. Since $(h-T h)^{\wedge}(n)=\left(1-e^{2 \pi i n \alpha}\right) \widehat{h}(n)$, a function $f$ in $B$ is a coboundary with transfer function in $B$ if and only if

$$
n \widehat{f}(n)=\mathrm{O}(\| n \alpha||) .
$$

If $f$ is the limit in $B$ of coboundaries $f_{j}$, then the sequence $(n \widehat{f}(n))$ is the uniform limit of $\left(n \widehat{f}_{j}(n)\right)$ and (6) follows. Conversely, suppose that for $\varepsilon>0$ there is an $\eta>0$ such that ||$n \alpha||<\eta$ implies $|n \widehat{f}(n)|<\varepsilon$; define $g_{\varepsilon} \in B$ by $\widehat{g}_{\varepsilon}(n)=\widehat{f}(n)$ when $\|n \alpha\| \geq \eta$ and $\widehat{g}_{\varepsilon}(n)=0$ otherwise. Then $\left\|f-g_{\varepsilon}\right\|_{B} \leq \varepsilon$ and $g_{\varepsilon}$ is a coboundary since

$$
\frac{\left|n \widehat{g}_{\varepsilon}(n)\right|}{\|n \alpha\|} \leq \frac{1}{\eta}\|f\|_{B}
$$

It follows from (6) that functions $f_{c}(x)=c\{x\}-c / 2, c \neq 0$, do not satisfy the mean ergodic theorem in $\mathrm{O}(1 / n)$ (since $\widehat{f}_{c}(n)=i c / n$ ) and the same result holds in $B V(\mathbf{T})$, since $B V(\mathbf{T}) \subset \mathrm{O}(1 / n)$. See Example 4 in the next subsection to see that they do not belong to the closure of coboundaries with measurable transfer functions.

The condition $h \in C$ from Corollary 3 cannot be strengthened to $h \in B$. Indeed if $f=g+h-T h$ with $g \in B_{h}$ and $h \in B$, since $B_{h}=\mathrm{o}(1 / n)$, we must have a constant $C$ such that for every sequence $n_{j} \rightarrow \infty$

$$
\left.\limsup \left|n_{j} \widehat{f}\left(n_{j}\right)\right|=\limsup \mid n_{j}(h-T h)^{\wedge}\left(n_{j}\right)\right) \mid \leq C \limsup \left\|n_{j} \alpha\right\|,
$$

and we can easily find $f$ satisfying (6) but not this latter condition. For example, let $f$ satisfy $\widehat{f}(n)=(1 / i n) \varphi(\{n \alpha\})$, where $\varphi$ is a bounded function on $\mathbf{T}$ such that $\varphi(x) \rightarrow 0$ but $\varphi(x) / x \rightarrow \infty$ as $x \rightarrow 0$.

In this example, we can choose for $\varphi$ a function with absolutely convergent Fourier series, so that $(\varphi(\{n \alpha\}))$ is the sequence of Fourier coefficients of a discrete measure concentrated on $\mathbf{Z} \alpha(\bmod 1)$. Then $f$ is a $B V$-function, which is not cohomologous to any $A C$-function (since $A C(\mathbf{T}) \subset \mathrm{o}(1 / n)$ ) via a transfer function in $\mathrm{O}(1 / n)$; we show below that such an $f$ belongs to $\operatorname{ET}(B V(\mathbf{T}), T)$.

Example 2. We consider now the case of $B V(\mathbf{T})$. Since we restrict to zero mean functions, we may identify $B V(\mathbf{T})$ with the space $M(\mathbf{T})$ of bounded real Borel measures on $\mathbf{T}$, equipped with the total variation norm. Then $T$ acts isometrically on $M(\mathbf{T})$ by $\mu \rightarrow \mu \circ T$ (the translation by $-\alpha$ ).

Proposition 5. A measure $\mu$ in $M(\mathbf{T})$ satisfies the mean ergodic theorem if and only if $\mu(A)=0$ for every $T$-invariant Borel set $A$ in $\mathbf{T}$. 
Proof. Let $\nu=\sum_{n=-\infty}^{+\infty} 2^{-|n|} T^{n}|\mu|$, and let $B$ be the subspace of all measures $\sigma$ in $M(\mathbf{T})$ with $|\sigma| \ll \nu$. Then $B$ is a closed $T$-invariant subspace of $M(\mathbf{T})$ containing $\mu$. Note that $\mu$ satisfies the mean ergodic theorem in $M(\mathbf{T})$ if and only if the same is true in $B$ and, by duality, if and only if $(h, \mu)=0$ for every $h \in B^{*}$ which is invariant under the adjoint map $T^{*}$.

Now $B$ is isomorphic to $L^{1}(\nu)$ and $T^{*}$ acts on $B^{*} \approx L^{\infty}(\nu)$ by the translation $h \rightarrow T h=h \circ T$. So $\mu \in \operatorname{ET}(M(\mathbf{T}), T)$ if and only if $\int h d \mu=0$ for any $T$-invariant element $h$ of $L^{\infty}(\nu)$.

Finally, if $h$ is a bounded Borel function with $h=T h \nu$-a.e., we also have $h=T^{n} h$ for every $n \in \mathbf{Z} \quad \nu$-a.e. (since $\nu$ is quasi-invariant under $T$ ) and we get a $T$-invariant Borel function equal to $h \nu$-a.e. by modifying $h$ on a set of zero $\nu$-measure. It follows that $\mu \in \operatorname{ET}(M(\mathbf{T}), T)$ if and only if $\int h d \mu=0$ for any $T$-invariant bounded Borel function $h$, which is clearly equivalent to $\mu(A)=0$ for every $T$-invariant Borel set $A$ in $\mathbf{T}$.

Suppose for example that $\mu$ is a discrete measure. Then for any $T$-invariant Borel set $A, \mu(A)$ is the sum of the measures of the cosets $x+\mathbf{Z} \alpha$ contained in $A$. Therefore, if $f$ is a jump function with bounded variation, the condition for $f \in \operatorname{ET}(B V(\mathbf{T}), T)$ is that the sum of its jumps on each coset of $\mathbf{Z} \alpha$ is zero.

Example 3. Suppose that $T:(X, \mathcal{B}, \mu) \longrightarrow(X, \mathcal{B}, \mu)$ is an ergodic automorphism on a standard Borel space. We will show that the characteristic function of a "typical" set $A \in \mathcal{B}$ does not satisfy the mean ergodic theorem in $L^{\infty}(X, \mu)$.

Fix $N \geq 1$. Notice that the family $\mathcal{A}_{N}$ of sets for which the first return time function is bounded by $N$ is closed. Moreover, $\mathcal{A}_{N}$ has empty interior (by removing a small set of positive measure we can easily increase the values of the first return time function). We conclude that the family $\mathcal{A}=\bigcup_{N>1} \mathcal{A}_{N}$ of sets with bounded first return time function forms a subset of first category in $\mathcal{B}$.

It remains to show that if $B \notin \mathcal{A}$ then $f=\chi_{B}-\mu(B)$ does not satisfy the mean ergodic theorem in $L^{\infty}(X, \mu)$. We can assume that $0<\mu(B)<1$. From our assumption there exists a strictly increasing sequence $\left(N_{i}\right)$ of natural numbers and a sequence $\left(B_{i}\right)$ of subsets of $B$ of positive measure such that if $x \in B_{i}$ then its first return time to $B$ is equal to $N_{i}$. For points $x \in B_{i}$

$$
\left|\frac{1}{N_{i}} \sum_{i=0}^{N_{i}-1} \chi_{B}\left(T^{i} x\right)-\mu(B)\right|=\left|1 / N_{i}-\mu(B)\right| .
$$

Therefore, $\frac{1}{n} \sum_{i=0}^{n-1} T^{i} f$ does not go to zero in $L^{\infty}(X, \mu)$ and the result follows.

When $X=\mathbf{T}$ and $T$ is an irrational translation, it is classical that Riemannintegrable functions satisfy the mean ergodic theorem. In [24], M. Talagrand shows that there are other functions which belong to $\operatorname{ET}\left(L^{\infty}(\mathbf{T}), T\right)$ for every irrational translation $T$ on $\mathbf{T}$. However, if $f$ is such a function, it follows from Theorem 2 that for each $1 \leq p<\infty$, there exists $h \in L^{p}(\mathbf{T})$ such that $f+h-T h$ is continuous.

In $\operatorname{Lip}(\mathbf{T})$ not all elements satisfy the mean ergodic theorem. Indeed, take $F(t)=\int_{0}^{t} f(x) d x$, where $f \in L^{\infty}(\mathbf{T})$ does not satisfy the mean ergodic theorem in $L^{\infty}(\mathbf{T})$.

2.2. Are the coboundaries dense in pseudo-homogeneous Banach spaces?

Let $B$ be a pseudo-homogeneous Banach space and $T: x \mapsto x+\alpha$ an irrational rotation on $\mathbf{T}$. We say that a Koksma inequality holds ([1]) for the pair $(B, T)$ provided that there exists a positive sequence $\widetilde{D}_{n}=\widetilde{D}_{n}(\alpha), n \geq 1$, satisfying 
$\widetilde{D}_{q_{n}}=\mathrm{O}\left(1 / q_{n}\right)$ where $\left(q_{n}\right)$ is the sequence of denominators of $\alpha$, such that for every $f \in B$

$$
\left\|\frac{1}{n} f^{(n)}-\int_{0}^{1} f(t) d t\right\|_{L^{1}} \leq\|f\|_{B} \tilde{D}_{n}(\alpha)
$$

where $f^{(N)}(x)=\sum_{j=0}^{N-1} f\left(T^{j} x\right), x \in \mathbf{T}$. For the classical cases where a Koksma inequality is satisfied for functions with bounded variation or Lipschitz continuous functions we refer to [11], chapter 2. In [1], a Koksma inequality has been proved for $B=\mathrm{O}(1 / n)$.

Similarly to [6], p.189, we have:

Proposition 6. If a Koksma inequality is satisfied for the pair $(B, T)$ then, for each $f \in B_{h}$ with zero mean, $\lim _{n \longrightarrow \infty} f^{\left(q_{n}\right)}=0$ in $L^{1}(\mathbf{T})$.

If $B$ is a homogeneous Banach space then coboundaries (even with transfer functions belonging to $B$ ) are dense. If $B$ is assumed to be a pseudo-homogeneous the situation is more complicated. It is well-known (in general setting even) that in $L^{\infty}(\mathbf{T})$ coboundaries with measurable transfer functions are dense (see e.g. [8]). As recently shown by $\mathrm{D}$. Volný [26], in $\operatorname{Lip}(\mathbf{T})$ coboundaries with measurable transfer functions are also dense. Notice that in general the closure of $B$-coboundaries is not contained in $B_{h}$ and this provides examples of pseudo-homogeneous spaces which are not homogeneous where coboundaries are dense.

That is generally not the case if $B$ satisfies a Koksma inequality. Set

$$
\mathcal{B}_{0}=\left\{f \in B: f^{\left(q_{n}\right)} \stackrel{\mu}{\longrightarrow} 0\right\} .
$$

Lemma 3. Assume that $B$ admits a Koksma inequality. Then $\mathcal{B}_{0}$ is closed and contains the closed subspace generated by coboundaries with measurable transfer functions which belong to $B$.

Proof. Let $f_{j} \rightarrow f$ in $B$ and $f_{j} \in \mathcal{B}_{0}$; then $\left\|f^{\left(q_{n}\right)}-f_{j}^{\left(q_{n}\right)}\right\|_{1} \leq$ const $\left\|f-f_{j}\right\|_{B}$, whence $f \rightarrow 0$ in measure. Besides, if $f=h-T h$, then $f^{\left(q_{n}\right)}=h-T^{q_{n}} h$ converges to zero in measure.

Now let $B$ be any pseudo-homogeneous space and let $f \in B$. Denote by $V_{f, T}$ the unitary operator on $L^{2}(\mathbf{T})$ defined by

$$
V_{f, T}(g)=e^{2 \pi i f} \cdot T g .
$$

Call the spectral measure $\sigma$ of the constant function 1 the spectral measure of $V_{f, T}$.

If $f \in \mathcal{B}_{0}$,

$$
\widehat{\sigma}\left[q_{n}\right]=\left(V_{f, T}^{q_{n}} 1,1\right)=\int_{0}^{1} e^{2 \pi i f^{\left(q_{n}\right)}} d \mu \longrightarrow 1 .
$$

A Borel probability measure $\nu$ on $\mathbf{T}$ is called a Dirichlet measure if $\widehat{\nu}\left[r_{n}\right] \longrightarrow 1$ for some sequence $\left(r_{n}\right)$. We obtain

Proposition 7. Assume that $B$ admits a Koksma inequality. If there exists $f \in B$ such that the spectral measure of $V_{f, T}$ is not a Dirichlet measure then coboundaries with measurable transfer functions are not dense in $B$.

Example 4. If $f(x)=f_{1}(x)=\{x\}-1 / 2$, the spectral measure of $V_{f, T}$ is Lebesgue measure, so it is not a Dirichlet measure (for an arbitrary irrational rotation). It follows that, in $B V(\mathbf{T})$ or $\mathrm{O}(1 / n), f_{1}$ and hence every $f_{c}$ with $c \neq 0$ do not belong to the closure of coboundaries with measurable transfer functions. 
Another example (even continuous) easily follows from [7] where for each irrational rotation by $\alpha$ (with $\alpha$ having unbounded partial quotients) a continuous function $f: \mathbf{T} \longrightarrow \mathbf{R}$ of bounded variation such that $T_{e^{2 \pi i f}}$ has Lebesgue spectrum (in the orthocomplement of the space of eigenfunctions) has been constructed.

\section{SpeCtral Approach to ERgodicity of CYlinder Flows}

In this section we will develop a method which will give us a link between spectral properties of $T_{\exp (2 \pi i f)}$ on $X \times \mathbf{T}$ and ergodicity of the cylinder flow $T_{f}$ on $X \times \mathbf{R}$.

We assume that $T$ is an ergodic automorphism, and $G$ is a locally compact second countable abelian group. Given a measurable function $f: X \longrightarrow G$, we denote $f_{*} \mu$ the direct image measure on $G$ defined by $f_{*} \mu(A)=\mu\left(f^{-1}(A)\right)$ for every Borel set $A$ in $G$. Let $\bar{G}$ denote the one-point compactification of $G$, and $\mathcal{P}(\bar{G})$ the set of Borel probability measures on $\bar{G}$. Recall that a sequence $\left(q_{n}\right)$ is said to be a rigidity time for $T$ if for each measurable function $f$ on $X, f \circ T^{q_{n}} \longrightarrow f$ in measure.

Proposition 8. Suppose that $f: X \longrightarrow G$ is a cocycle. Let $\left(q_{n}\right)$ be a rigid sequence for $T$ and let $W$ be a compact subset of $G$. If

$$
\inf _{n \geq 1} \mu\left(\left[f^{\left(q_{n}\right)} \in W\right]\right)>0
$$

then

$$
E(f) \cap W \neq \emptyset .
$$

In particular, if in addition $0 \notin W$ then $f$ is not of type $I I I_{0}$.

Proof. Consider the measures $\left(f^{\left(q_{n}\right)}\right)_{*} \mu$ as probability measures on $\bar{G}$. By weak compactness of $\mathcal{P}(\bar{G})$, we can assume that this sequence converges weakly to some $\nu \in \mathcal{P}(\bar{G})$. Then $\nu(W)>0$, and all we need to show is that $\operatorname{supp}(\nu) \subset \bar{E}(f)$.

First we claim that for each $\varphi \in C(\bar{G})$ and each $g \in L^{1}(X, \mu)$,

$$
\int_{X} \varphi \circ f^{\left(q_{n}\right)} g d \mu \longrightarrow \int_{\bar{G}} \varphi d \nu \int_{X} g d \mu .
$$

As $\left(f^{\left(q_{n}\right)}\right)_{*} \mu \rightarrow \nu$ weakly, (7) holds for constant functions $g$ and thus it is enough to prove that the limit is 0 when $\int_{X} g d \mu=0$. Moreover, since coboundaries are dense in the subspace of functions with zero mean, we can restrict to the case $g=h-T h$, $h \in L^{1}(X, \mu)$. Then

$$
\begin{aligned}
\int_{X} \varphi \circ f^{\left(q_{n}\right)} g d \mu & =\int_{X} \varphi \circ f^{\left(q_{n}\right)} h d \mu-\int_{X} \varphi \circ f^{\left(q_{n}\right)} T h d \mu \\
& =\int_{X} \varphi\left(f^{\left(q_{n}\right)}(T x)\right) h(T x) d \mu(x)-\int_{X} \varphi\left(f^{\left(q_{n}\right)}(x)\right) h(T x) d \mu(x) \\
& =\int_{X}\left(\varphi\left(f^{\left(q_{n}\right)}(T x)\right)-\varphi\left(f^{\left(q_{n}\right)}(x)\right)\right) h(T x) d \mu(x) .
\end{aligned}
$$

Since $T f^{\left(q_{n}\right)}-f^{\left(q_{n}\right)}=T^{q_{n}} f-f$ and $\left(q_{n}\right)$ is a rigidity time for $T, T f^{\left(q_{n}\right)}-f^{\left(q_{n}\right)}$ converges to 0 in measure; as $\varphi$ is uniformly continuous and bounded, $\varphi\left(f^{\left(q_{n}\right)}(T x)\right)-$ $\varphi\left(f^{\left(q_{n}\right)}(x)\right)$ still converges to 0 in measure and the integral goes to 0 . Hence the claim is established.

Now let $B \in \mathcal{B}$ be a set of positive measure, $r \in \operatorname{supp}(\nu)$ and $U$ a neighbourhood of $r$ in $\bar{G}$. Choose a continuous function $\varphi$ on $\bar{G}$ with $0 \leq \varphi \leq \chi_{U}$ and $\int_{\bar{G}} \varphi d \nu>0$. 
Then $\mu\left(B \cap\left[f^{\left(q_{n}\right)} \in U\right]\right) \geq \int_{B} \varphi \circ f^{\left(q_{n}\right)} d \mu$; it follows from (7), with $g=\chi_{B}$, that

$$
\liminf _{n \rightarrow \infty} \mu\left(B \cap\left[f^{\left(q_{n}\right)} \in U\right]\right) \geq \mu(B) \int_{\bar{G}} \varphi d \nu>0
$$

whence, since $q_{n}$ is a rigid sequence for $T$,

$$
\liminf _{n \longrightarrow \infty} \mu\left(B \cap T^{-q_{n}} B \cap\left[f^{\left(q_{n}\right)} \in U\right]\right)>0 .
$$

This proof actually shows more precisely the following:

Proposition 9. Suppose that $f: X \longrightarrow G$ is a cocycle. Let $\left(q_{n}\right)$ be a rigid sequence for $T$ and suppose that $\left(f^{\left(q_{n}\right)}\right)_{*} \mu \longrightarrow \nu$ weakly in $\mathcal{P}(\bar{G})$. Then

$$
\operatorname{supp}(\nu) \subset \bar{E}(f) .
$$

Remark 1 . We obtain a necessary condition for $f$ to be of type $I I I_{0}$, namely:

$$
\text { for each rigid sequence }\left(q_{n}\right) \text { for } T,\left\{\left(f^{\left(q_{n}\right)}\right)_{*} \mu\right\}^{\prime} \subset \mathcal{P}(\{0, \infty\})
$$

where $\left\{\left(f^{\left(q_{n}\right)}\right)_{*} \mu\right\}^{\prime}$ denotes the set of weak limit points of $\left(\left(f^{\left(q_{n}\right)}\right)_{*} \mu\right)$ in $\mathcal{P}(\bar{G})$.

The following proposition is a generalization of a well-known fact for the compact group case (see [22], [25] for the case of irrational rotations and circle cocycles, [12] for arbitrary rotations and Abelian groups and [4] for the most general case).

Proposition 10. Suppose $T: X \longrightarrow X$ is an ergodic rotation on a compact group and $f: X \longrightarrow G$ is measurable such that

$$
f^{(n)} \stackrel{\mu}{\longrightarrow} 0 \text { as } \quad T^{n} \longrightarrow I d
$$

then $f$ is a coboundary.

Proof. Let $\varepsilon>0$; then by assumption there exists a neighbourhood $U \subset X, 0 \in U$ such that

$$
T^{n} \in U \Rightarrow \mu\left(\left[\left|f^{(n)}\right| \geq 1\right]\right)<\varepsilon .
$$

By minimality of $T: X \longrightarrow X$, we have that $K_{U}:=\left\{n \in \mathbf{Z}: T^{n} \in U\right\}$ has bounded gaps, and hence there exists a finite set $F \subset \mathbf{N}$ such that

$$
F+K_{U}=\mathbf{Z} .
$$

Then every integer $n$ may be written $n=j+k$ where $j \in F$ and $k \in K_{U}$; since there clearly exists $M>0$ such that $\mu\left(\left[\left|f^{(j)}\right| \geq M\right]\right)<\varepsilon$ for all $j \in F$, we have

$$
\begin{aligned}
\mu\left(\left[\left|f^{(n)}\right| \geq M+1\right]\right) & =\mu\left(\left[\left|f^{(j)}+f^{(k)} \circ T^{j}\right| \geq M+1\right]\right) \\
& \leq \mu\left(\left[\left|f^{(j)}\right| \geq M\right]\right)+\mu\left(\left[\left|f^{(k)}\right| \geq 1\right]\right)<2 \varepsilon
\end{aligned}
$$

and by [16], $f$ must be a coboundary.

Suppose the condition (8) holds. It is tempting to expect that the existence of a rigid sequence $\left(q_{n}\right)$ for $T$ such that

$$
\left(f^{q_{n}}\right)_{*} \mu \rightarrow \nu \neq \delta_{0}
$$

implies that $f$ is of type $I I I_{0}$. Somewhat surprisingly this is not the case. As noticed in [2], squashable cocycles satisfy (8) and there exist ergodic squashable cocycles. 
Proposition 11. Let $T:(X, \mathcal{B}, \mu) \longrightarrow(X, \mathcal{B}, \mu)$ be ergodic. Assume that $G, H$ are locally compact abelian groups and let $h: G \longrightarrow H$ be a continuous group homomorphism. Let $f: X \longrightarrow G$ be a cocycle. Then

$$
\overline{h(E(f))} \subset E(h \circ f) \text {. }
$$

If $f$ is regular then

$$
\overline{h(E(f))}=E(h \circ f) .
$$

Proof. Let $r \in E(f)$ and $U$ be a neighbourhood of the unit of $G$. Take $B \in$ $\mathcal{B}, \mu(B)>0$. Then there exists $N \in \mathbf{Z}$ such that

$$
\mu\left(B \cap T^{-N} B \cap\left[f^{(N)} \in h^{-1}(U)+r\right]\right)>0 .
$$

Since $h \circ f^{(N)}=(h \circ f)^{(N)}$, we have

$$
B \cap T^{-N} B \cap\left[f^{(N)} \in h^{-1}(U)+r\right] \subset B \cap T^{-N} B \cap\left[(h \circ f)^{(N)} \in U+h(r)\right]
$$

and therefore $h(r) \in E(h \circ f)$. Since $E(h \circ f)$ is closed, the first part is proved.

If $f$ is regular then $f=g+j-T j$ where $g: X \longrightarrow E(f)$ (see [23], part I). So $h \circ f$ is cohomologous to $h \circ g$ and $E(h \circ f)=E(h \circ g) \subset \overline{h(E(f))}$ since $h \circ g: X \longrightarrow \overline{h(E(f))}$ and the latter group is closed.

Remark 2. If $f$ is of type $I I I_{0}$, then in general there is no equality since $h \circ f$ can be even ergodic. Note that if $G=\mathbf{R}$ and $E(f)=r \mathbf{Z}$ where $r \notin \mathbf{Q}$, then $e^{2 \pi i f}: X \longrightarrow \mathbf{T}$ is an ergodic cocycle.

3.1. Spectral criteria for ergodicity of cylinder flows. In this subsection $T$ stands for an ergodic automorphism of $(X, \mathcal{B}, \mu)$. Let $\nu$ be a probability measure on $\mathbf{R}$. By $\tilde{\nu}$ we will mean the measure $(\exp 2 \pi i \cdot)_{*} \nu$. Put

$$
\widehat{\tilde{\nu}}[k]=\int_{0}^{1} e^{2 \pi i k x} d \tilde{\nu}(x) .
$$

Suppose that $f_{n}: X \longrightarrow \mathbf{R}, n \geq 1$, are measurable and set

$$
\mu_{n}=\left(f_{n}\right)_{*} \mu, \quad n \geq 1 .
$$

If $\mu_{n} \longrightarrow \nu$ weakly in $\mathcal{P}(\overline{\mathbf{R}})$ then, since $\nu$ is concentrated on $\mathbf{R}, \tilde{\mu}_{n}$ converges weakly to $\tilde{\nu}$. Consequently, in this case $\widehat{\tilde{\mu}}_{n}[k] \longrightarrow \widehat{\hat{\nu}}[k]$ for all $k \in \mathbf{Z}$. But $\widehat{\tilde{\mu}}_{n}[k]=$ $\int_{X} e^{2 \pi i k f_{n}(x)} d \mu(x)$ whence

$$
\int_{X} e^{2 \pi i k f_{n}} d \mu \longrightarrow \widehat{\tilde{\nu}}[k]
$$

for all $k \in \mathbf{Z}$. We will need also the following simple

Lemma 4. If $\nu$ is discrete, then $\tilde{\nu}$ is discrete and in particular $\tilde{\nu}$ is a Dirichlet measure.

Proof. If $\nu$ is concentrated on a countable set $D$, then $\tilde{\nu}$ is concentrated on $\left\{e^{2 \pi i r}\right.$; $r \in D\}$ and $\widehat{\tilde{\nu}}\left[k_{j}\right] \rightarrow 1$ for every sequence $\left(k_{j}\right)$ such that $k_{j} r \rightarrow 0 \bmod 1$ for every $r \in D$.

Now let $f: X \longrightarrow \mathbf{R}$ be measurable and $\left(q_{n}\right)$ be a rigid sequence for $T$. Assume

$$
\sup _{n} \mu\left\{x \in X:\left|f^{\left(q_{n}\right)}\right|>A\right\} \longrightarrow 0 \text { when } A \longrightarrow \infty .
$$

This condition will be satisfied if for example $f \in L^{1}(X, \mu)$ and $\left(\left\|f^{\left(q_{n}\right)}\right\|_{L^{1}}\right)$ is bounded. 
Then put $\mu_{n}=\left(f^{\left(q_{n}\right)}\right)_{*} \mu$. By considering $\left(\mu_{n}\right)$ as a sequence of measures on $\overline{\mathbf{R}}$ we can choose a subsequence, still denoted by $\left(\mu_{n}\right)$, which converges weakly towards some probability measure $\nu$. Because of (10) we have then $\nu(\{\infty\})=0$, so $\nu$ is concentrated on $\mathbf{R}$. In view of (9),

$$
\int_{X} e^{2 \pi i k f^{\left(q_{n}\right)}} d \mu \longrightarrow \widehat{\widetilde{\nu}}[k]
$$

for all $k \in \mathbf{Z}$.

Proposition 12. Suppose $f: X \rightarrow \mathbf{R}$ is measurable and satisfies (10). If

$$
\limsup _{n \rightarrow \infty}\left|\int_{X} e^{2 \pi i k f^{\left(q_{n}\right)}} d \mu\right| \leq c<1 \text { for all } k \text { large enough, }
$$

then $T_{f}$ is ergodic.

Proof. If $T_{f}$ is not ergodic, we have $E(f)=r \mathbf{Z}$ for some $r \in \mathbf{R}$. Then any weak limit $\nu$ of a subsequence of $\left(\left(f^{\left(q_{n}\right)}\right)_{*} \mu\right)$ is concentrated on $r \mathbf{Z}$. Now, by (11), we have $\widehat{\tilde{\nu}}[k] \leq c<1$ for all $k$ large enough, which contradicts Lemma 4 .

Consider now $T_{e^{2 \pi i f}}:(X \times \mathbf{T}, \mu \times \lambda) \longrightarrow(X \times \mathbf{T}, \mu \times \lambda)$. We have that

$$
L^{2}(X \times \mathbf{T}, \mu \times \lambda)=\bigoplus_{k \in \mathbf{Z}} L_{k}
$$

where

$$
L_{k}=\left\{F(x, y)=f(x) e^{2 \pi i k y}: f \in L^{2}(X, \mu)\right\} .
$$

The spaces $L_{k}$ are closed and $T_{e^{2 \pi i f}}$-invariant. Moreover, the action of $T_{e^{2 \pi i f}}$ on $L_{k}$ is unitarily equivalent to the action of $V_{k}: L^{2}(X, \mu) \longrightarrow L^{2}(X, \mu)$ given by $\left(V_{k} g\right)(x)=e^{2 \pi i k f(x)} g(T x)$. Notice finally that given $k, s \in \mathbf{Z}$ we have

$$
\int_{X} e^{2 \pi i k f^{(s)}(x)} d \mu(x)=\widehat{\sigma}_{k}[s]
$$

where $\sigma_{k}$ denotes the spectral measure of the constant function 1 under $V_{k}$.

Proposition 13. If $f$ satisfies (10) with $\left(q_{n}\right)$ a rigidity time for $T$ and the maximal spectral type $\sigma$ of $T_{e^{2 \pi i f}}$ on $\bigoplus_{k \neq 0} L_{k}$ is a Rajchman measure (i.e. $\widehat{\sigma}$ vanishes at infinity), then $E(f)=\mathbf{R}$ and $T_{f}$ is ergodic. In particular, $T_{f}$ is ergodic whenever $T_{e^{2 \pi i f}}$ has Lebesgue spectrum in the orthocomplement of $L_{0}$.

Proof. For all $k \neq 0$, since $\sigma_{k} \ll \sigma$ we know by the so-called generalized RiemannLebesgue lemma that $\sigma_{k}$ is also a Rajchman measure, i.e. $\widehat{\sigma}_{k}[s] \rightarrow 0$ when $s \rightarrow \infty$. In particular

$$
\widehat{\sigma}_{k}\left[q_{n}\right]=\int_{X} e^{2 \pi i k f^{\left(q_{n}\right)}} d \mu \longrightarrow 0 .
$$

We conclude by Proposition 12.

Remark 3. Notice that under the hypothesis of Proposition 13, if $\nu$ is a weak limit point of $\left(\left(f^{\left(q_{n}\right)}\right)_{*} \mu\right)$, by (11) $\tilde{\nu}$ is the Lebesgue measure. Then necessarily $\nu$ itself is absolutely continuous (since $\exp (2 \pi i \cdot): \mathbf{R} \rightarrow \mathbf{T}$ is non-singular). 
3.2. Applications for irrational rotations. Suppose now that $T x=x+\alpha$ is an irrational rotation and $\left(q_{n}\right)$ is the sequence of denominators of $\alpha$ (but we shall consider subsequences without changing the notation). If a Koksma inequality holds for a pseudo-homogeneous space $B$ then any $f$ in $B$ (with zero mean) satisfies condition (10), since $\left(\left\|f^{\left(q_{n}\right)}\right\|_{1}\right)$ is bounded and it follows that any weak limit of $\left(f^{\left(q_{n}\right)}\right)_{*} \lambda$ in $\mathcal{P}(\overline{\mathbf{R}})$ is concentrated on $\mathbf{R}$.

Recall that $\mathcal{B}_{0}$ denotes the subspace of all $f \in B$ such that $f^{\left(q_{n}\right)} \rightarrow 0$ in measure. As a consequence of Proposition 9 we have:

Corollary 4. Let $B \subset L^{1}(\mathbf{T})$ be a pseudo-homogeneous space and suppose that $B$ admits a Koksma inequality. Assume that $f \in B \backslash \mathcal{B}_{0}$. Then $f$ is not of type $I I I_{0}$ (and hence $f$ is regular).

Proof. Since $f^{\left(q_{n}\right)}$ does not converge to zero in measure, we can find $\left(n_{k}\right)$ such that $f_{*}^{\left(q_{n_{k}}\right)} \lambda \rightarrow \nu$ in $\mathcal{P}(\overline{\mathbf{R}})$, where $\nu(\{0\})<1$. Then $\nu \notin \mathcal{P}(\{0, \infty\})$, since $\nu$ is concentrated on $\mathbf{R}$. We conclude by Remark 1 .

Given $B$ let us denote

$$
\operatorname{Erg}(B, T)=\left\{f \in B: T_{f} \text { is ergodic }\right\} .
$$

Directly from Proposition 13 we obtain the following:

Proposition 14. Let $B$ be a pseudo-homogeneous Banach space with a Koksma inequality and let $f \in B$. If $f$ satisfies (12) then $f \in \operatorname{Erg}(B, T)$. In particular, if the maximal spectral type of $T_{e^{2 \pi i f}}$ in the orthocomplement of $L_{0}$ is a Rajchman measure, then $f \in \operatorname{Erg}(B, T)$.

Corollary 5. Let $f$ be a function in $L^{1}(\mathbf{T})$ with zero mean. If the Fourier coefficients of $f$ are of order $\mathrm{O}(1 / n)$ and $T_{e^{2 \pi i f}}$ has Lebesgue spectrum in the orthocomplement of $L_{0}$ then $f \in \operatorname{Erg}(B, T)$.

Now our spectral approach offers more, namely stability properties for ergodicity of cocycles which we shall study in detail for bounded variation functions in the next section. We begin by some results for general pseudo-homogeneous spaces with a Koksma inequality.

It is clear that if $f \in \operatorname{Erg}(B, T)$, then $f+g \in \operatorname{Erg}(B, T)$ for every coboundary $g=h-T h \in B$. Suppose that $f$ moreover satisfies (12). If $g \in B$ and

$$
\left|\int_{\mathbf{T}} e^{2 \pi i g^{\left(q_{n}\right)}} d \lambda\right| \rightarrow 1
$$

then there exists a sequence of constants $c_{n}$ of modulus 1 such that $c_{n} e^{2 \pi i g^{\left(q_{n}\right)}} \rightarrow 1$ in measure. This implies

$$
\limsup _{n \rightarrow \infty}\left|\int_{\mathbf{T}} e^{2 \pi i k\left(f^{\left(q_{n}\right)}+g^{\left(q_{n}\right)}\right)} d \lambda\right|=\limsup _{n \rightarrow \infty}\left|\int_{\mathbf{T}} e^{2 \pi i k f^{\left(q_{n}\right)}} d \lambda\right|
$$

for every $k$, and it follows that $f+g \in \operatorname{Erg}(B, T)$.

Now, (13) holds clearly if $g \in B_{0}$. This is also the case if $e^{2 \pi i g}$ is a circle quasi-coboundary, i.e.

$$
e^{2 \pi i g(x)}=c \frac{\eta\left(e^{2 \pi i T x}\right)}{\eta\left(e^{2 \pi i x}\right)}
$$

where $|c|=1$ and $\eta: \mathbf{T} \rightarrow \mathbf{T}$ is measurable. We have shown: 
Proposition 15. Let $B$ be a pseudo-homogeneous Banach space with a Koksma inequality and let $f, g \in B$. If $f$ satisfies (12) and either $g \in B_{0}$ or $e^{2 \pi i g}$ is a circle quasi-coboundary, then $f+g \in \operatorname{Erg}(B, T)$.

We have a similar result for every $g \in B \backslash \operatorname{Erg}(B, T)$. We need an auxiliary lemma:

Lemma 5. Let $f_{n}$ and $g_{n}: \mathbf{T} \rightarrow \mathbf{R}, n \geq 1$, be measurable. Assume that $\left(f_{n}\right)_{*} \lambda \rightarrow$ $\nu,\left(g_{n}\right)_{*} \lambda \rightarrow \sigma$ and $\left(f_{n}+g_{n}\right)_{*} \lambda \rightarrow \tau$ weakly in $\mathcal{P}(\overline{\mathbf{R}})$, where $\nu, \sigma$ and $\tau$ are concentrated on $\mathbf{R}$ and $\sigma$ is concentrated on $r \mathbf{Z}$ for some $r \in \mathbf{R}$.

Then if $\nu$ is not discrete, then $\tau$ is not discrete.

Proof. We may assume $r \neq 0$ and replace $f$ and $g$ by $\frac{1}{r} f$ and $\frac{1}{r} g$; hence we may assume $r=1$.

Then $\sigma$ is a probability measure concentrated on $\mathbf{Z}$ and $\tilde{\sigma}$ is the Dirac measure at 1 . It follows that $e^{2 \pi i k g_{n}}$ converges to 1 in measure. Therefore

$$
\widehat{\tilde{\tau}}(k)=\lim _{n \rightarrow \infty} \int_{\mathbf{T}} e^{2 \pi i k\left(f_{n}+g_{n}\right)} d \lambda=\lim _{n \rightarrow \infty} \int_{\mathbf{T}} e^{2 \pi i k f_{n}} d \lambda=\widehat{\tilde{\nu}}(k)
$$

for every $k$, whence $\tilde{\tau}=\tilde{\nu}$. Now $\tilde{\nu}$ is not discrete since $\nu$ is not discrete, and it follows that $\tau$ is not discrete.

Proposition 16. Let $B$ be a pseudo-homogeneous Banach space with a Koksma inequality and let $f, g \in B$. If $f$ satisfies (12) and $g \notin \operatorname{Erg}(B, T)$, then $f+g \in$ $\operatorname{Erg}(B, T)$.

Proof. By taking a subsequence, we may assume that

$$
\left(f^{\left(q_{n}\right)}\right)_{*} \lambda, \quad\left(g^{\left(q_{n}\right)}\right)_{*} \lambda \quad \text { and } \quad\left(f^{\left(q_{n}\right)}+g^{\left(q_{n}\right)}\right)_{*} \lambda
$$

converge weakly towards probability measures $\nu, \sigma$ and $\tau$. As $B$ admits a Koksma inequality, those measures are concentrated on $\mathbf{R}$. Moreover $\nu$ is not discrete since $f$ satisfies (12) and $\sigma$ is concentrated on some subgroup $r \mathbf{Z}$ since $T_{g}$ is not ergodic. We conclude by Lemma 5 that $\tau$ is not discrete, whence $T_{f+g}$ is ergodic.

Remark 4. The same result holds if some weak limit point of $\left(\left(f^{\left(q_{n}\right)}\right)_{*} \lambda\right)$ is not discrete. Note that in this proof, we obtain that $\tau$ is not discrete. It follows that if $f$ satisfies (12) and $g_{1}, \ldots, g_{k} \in B \backslash \operatorname{Erg}(B, T)$, then $f+g_{1}+\cdots+g_{k} \in \operatorname{Erg}(B, T)$.

Besides, the same proof shows that if $\left(f^{\left(q_{n}\right)}\right)_{*} \lambda \longrightarrow \nu$ where $\nu$ is absolutely continuous and $g$ is not ergodic, then each limit point of $\left(f^{\left(q_{n}\right)}+g^{\left(q_{n}\right)}\right)_{*} \lambda$ is absolutely continuous.

\section{Functions of bounded variation. The structure OF ERGODIC COYCLES}

This section will be devoted to a detailed study of ergodicity of cocycles of bounded variation. We have already seen that measurable coboundaries are not dense in $B V(\mathbf{T})$ (Example 4). We will now show that even more happens. Namely, there are ergodic cocycles in $B V(\mathbf{T})$ such that any sufficiently close cocycle remains ergodic. We will call this property ergodic stability. In particular we show that straight lines are ergodically stable. 
Definition. A function $f:[0,1) \longrightarrow \mathbf{R}$ is said to be piecewise absolutely continuous (PAC) if there are $y_{1}, \ldots, y_{K} \in[0,1)$ such that $\left.f\right|_{\left(y_{i}, y_{i+1}\right)}$ is absolutely continuous with limits

$$
f_{+}\left(y_{i}\right)=\lim _{x \longrightarrow y_{i}^{+}} f(x) \text { and } f_{-}\left(y_{i}\right)=\lim _{x \longrightarrow y_{i}^{-}} f(x)
$$

for $i=1, \ldots, K\left(y_{K+1}=y_{1}\right)$.

Obviously, each $f$ which is PAC is of bounded variation. Denote

$$
S(f)=\int_{0}^{1} f^{\prime}(t) d \lambda(t)=\sum_{j=1}^{K} f_{-}\left(y_{i}\right)-f_{+}\left(y_{i}\right) .
$$

We will say that $f$ is strongly PAC (SPAC) if in addition $f^{\prime}$ has bounded variation.

Theorem 3. Suppose that $f$ is $P A C$ and that $S(f) \neq 0$. Then $f$ is ergodically stable in $B V(\mathbf{T})$. More precisely, if $g \in B V(\mathbf{T})$ and $\operatorname{Var}(f-g)<|S(f)|$, then $g$ is still ergodic.

Proof. Let $S=|S(f)|$. We shall prove that if $g \in B V(\mathbf{T})$, $\operatorname{Var}(g)<S$ and $c$ is any constant with $\operatorname{Var}(g) / S<c<1$, then

$$
\limsup _{n \rightarrow \infty}\left|\int_{\mathbf{T}} e^{2 \pi i k\left(f^{\left(q_{n}\right)}+g^{\left(q_{n}\right)}\right)} d \lambda\right| \leq c
$$

for every $k$ large enough. By Proposition 14 this implies that $f+g$ is ergodic.

Notice first that each $f$ which is PAC can be represented as $f=f_{0}+h$, where $f_{0}$ is SPAC (even piecewise linear) with $S\left(f_{0}\right)=S(f)$ and $h \in A C(\mathbf{T})$. Then $h^{\left(q_{n}\right)} \longrightarrow 0$ in measure by Proposition 6 , whence $\left(f^{\left(q_{n}\right)}+g^{\left(q_{n}\right)}\right)_{*} \lambda$ and $\left(f_{0}^{\left(q_{n}\right)}+g^{\left(q_{n}\right)}\right)_{*} \lambda$ have the same weak limit points in $\mathcal{P}(\overline{\mathbf{R}})$ and the limit in (15) is the same if we replace $f$ by $f_{0}$. So, we can restrict ourselves to the case when $f$ is SPAC.

Then let

$$
I_{k, q}=\int_{\mathbf{T}} e^{2 \pi i k\left(f^{(q)}+g^{(q)}\right)} d \lambda
$$

for $k \neq 0$ and $q \geq 1$.

Suppose $\operatorname{Var}(g) / S<c<1$ and choose $\varepsilon>0$ such that $\operatorname{Var}(g) /(S-\varepsilon)<c$. Since $f^{\prime}$ has bounded variation and thus $\frac{1}{q}\left|f^{\prime(q)}\right| \longrightarrow S$ uniformly, we have for $q$ large enough, say $q \geq q_{0}$,

$$
\left|f^{\prime(q)}(x)\right| \geq(S-\varepsilon) q \text { for all } x \in \mathbf{T} .
$$

We henceforth suppose that $q \geq q_{0}$.

Denote by $x_{1} \leq x_{2} \leq \ldots \leq x_{q K}$ the sequence of all points of the form $\left(y_{j}-s \alpha\right)$ for $j=1, \ldots, K$ and $s=0,1, \ldots, q-1$ ordered increasingly. Divide [0,1) into subintervals $\left[x_{j}, x_{j+1}\right), j=1, \ldots, q K\left(x_{q K+1}=x_{1}\right)$. It may happen that some of these intervals are in fact empty. However, if $\left[x_{j}, x_{j+1}\right)$ is not degenerated then $\left.f^{(q)}\right|_{\left(x_{j}, x_{j+1}\right)}$ is absolutely continuous and $\left(f^{(q)}\right)^{\prime}(x)=f^{\prime(q)}(x)$ for $x \in\left(x_{j}, x_{j+1}\right)$. We have then

$$
\int_{x_{j}}^{x_{j+1}} e^{2 \pi i k\left(f^{(q)}+g^{(q)}\right)} d \lambda=\int_{x_{j}}^{x_{j+1}} \frac{e^{2 \pi i k g^{(q)}}}{2 \pi i k f^{\prime(q)}} d\left(e^{2 \pi i k f^{(q)}}\right) .
$$


Since $f^{\prime(q)}$ has bounded variation, we obtain by integrating by parts on each interval $\left(x_{j}, x_{j+1}\right)$

$$
\begin{aligned}
I_{k, q}= & \frac{1}{2 \pi i k} \sum_{j=1}^{K q}\left(\frac{e^{2 \pi i k\left(f_{-}^{(q)}\left(x_{j+1}\right)+g_{-}^{(q)}\left(x_{j+1}\right)\right)}}{f_{{ }_{-}^{\prime}(q)}\left(x_{j+1}\right)}-\frac{e^{2 \pi i\left(k f_{+}^{(q)}\left(x_{j}\right)+k g_{+}^{(q)}\left(x_{j}\right)\right)}}{f_{+}^{\prime(q)}\left(x_{j}\right)}\right) \\
& -\frac{1}{2 \pi i k} \int_{0}^{1} e^{2 \pi i k f^{(q)}} d\left(e^{2 \pi i k g^{(q)}} / f^{\prime(q)}\right) .
\end{aligned}
$$

According to (16), it follows that

$$
\left|I_{k, q}\right| \leq \frac{1}{2 \pi|k|}\left(\frac{2 K q}{(S-\varepsilon) q}+\operatorname{Var}\left(e^{2 \pi i k g^{(q)}} / f^{\prime(q)}\right)\right)
$$

and

$$
\begin{aligned}
\operatorname{Var}\left(e^{2 \pi i k g^{(q)}} / f^{\prime(q)}\right) & \leq \sup \left(1 /\left|f^{\prime(q)}\right|\right) \operatorname{Var}\left(e^{2 \pi i k g^{(q)}}\right)+\operatorname{Var}\left(1 / f^{\prime(q)}\right) \\
& \leq \frac{1}{(S-\varepsilon) q} 2 \pi|k| \operatorname{Var}\left(g^{(q)}\right)+\frac{1}{(S-\varepsilon)^{2} q^{2}} \operatorname{Var}\left(f^{\prime(q)}\right) \\
& \leq \frac{2 \pi|k|}{S-\varepsilon} \operatorname{Var}(g)+\frac{1}{(S-\varepsilon)^{2} q} \operatorname{Var}\left(f^{\prime}\right) .
\end{aligned}
$$

Finally

$$
\left|I_{k, q}\right| \leq \frac{K}{\pi|k|(S-\varepsilon)}+\frac{\operatorname{Var}(g)}{S-\varepsilon}+\frac{\operatorname{Var}\left(f^{\prime}\right)}{2 \pi|k|(S-\varepsilon)^{2} q}=\frac{\operatorname{Var}(g)}{S-\varepsilon}+\mathrm{O}(1 / k)
$$

uniformly in $q \geq q_{0}$. Since $\operatorname{Var}(g) /(S-\varepsilon)<c$, we have $\lim \sup _{q \rightarrow \infty}\left|I_{k, q}\right| \leq c$ for all $k$ large enough.

Using the method of this proof one can obtain more information about limit points of $\left(\left(f^{\left(q_{n}\right)}\right)_{*} \lambda\right)$.

Corollary 6. If $f$ is PAC, $S(f) \neq 0$ and $\left(f^{\left(q_{n}\right)}\right)_{*} \lambda \longrightarrow \nu$ then $\nu$ is absolutely continuous.

Proof. In the above proof, when $g=0$, we obtain that $\widehat{\tilde{\nu}}[k]=\lim \int e^{2 \pi i k f^{\left(q_{n}\right)}} d \lambda=$ $\mathrm{O}(1 / k)$, whence $\tilde{\nu}$ is absolutely continuous.

As a direct consequence of Theorem 3 we obtain

Corollary 7. $\operatorname{Int}(\operatorname{Erg}(B V(\mathbf{T}), T)) \neq \emptyset$.

In fact we have more:

Corollary 8. Suppose $f$ is $P A C, S(f) \neq 0$, and $g \in B V(\mathbf{T})$. If either $g \notin$ $\operatorname{Erg}(B V(\mathbf{T}), T)$, or $g \in B V(\mathbf{T})_{0}$, or $e^{2 \pi i g}$ is a quasi-coboundary, then $f+g$ is ergodically stable.

Proof. Let $h \in B V(\mathbf{T})$ with $\operatorname{Var}(h)<|S(f)|$. In the proof of Theorem 3 we have actually shown that under this assumption $f+h$ satisfies (12). So $f+g+$ $h \in \operatorname{Erg}(B V(\mathbf{T}), T)$ follows from Proposition 16 if $g \notin \operatorname{Erg}(B V(\mathbf{T}), T)$ and from Proposition 15 in both other cases.

Corollary 9. $\operatorname{Int}(\operatorname{Erg}(B V(\mathbf{T}), T))$ is dense in $B V(\mathbf{T})$. 
Proof. Let $g \in B V(\mathbf{T})$ and suppose first that $T_{g}$ is not ergodic. Let $f$ be PAC, with $S(f) \neq 0$. By Corollary $8, g+\delta f$ is ergodically stable for every $\delta>0$; so $g$ can be approximated by cocycles in $\operatorname{Int}(\operatorname{Erg}(B V(\mathbf{T}), T))$. Now if $g \in \operatorname{Erg}(B V(\mathbf{T}), T)$ then either $g$ belongs to $\operatorname{Int}(\operatorname{Erg}(B V(\mathbf{T}), T))$, or it can be approximated by non-ergodic cocycles and therefore by cocycles in $\operatorname{Int}(\operatorname{Erg}(B V(\mathbf{T}), T))$.

Remark 5. Corollary 8 gives us a possibility to show that if $\alpha$ has unbounded partial quotients, then there are some continuous ergodically stable cocycles. Indeed in [7] one constructs a continuous cocycle $\xi: \mathbf{T} \rightarrow \mathbf{T}$ of bounded variation homotopic to the identity and such that $\xi$ is a circle coboundary:

$$
\xi=\eta\left(e^{2 \pi i T x}\right) / \eta\left(e^{2 \pi i x}\right) .
$$

Since $\xi$ is homotopic to the identity, there exist $f \in B V(\mathbf{T})$ continuous with zero mean and a constant $A$ such that

$$
\xi\left(e^{2 \pi i x}\right)=\exp 2 \pi i(f(x)+x+A) .
$$

Then, if $g(x)=f(x)+x-\frac{1}{2}$,

$$
e^{2 \pi i g(x)}=e^{-2 \pi i(A+1 / 2)} \eta\left(e^{2 \pi i(x+\alpha)}\right) / \eta\left(e^{2 \pi i x}\right),
$$

so $g$ is a quasi-coboundary, and by Corollary 8

$$
f(x)=g(x)+x-\frac{1}{2} \in \operatorname{Int}(\operatorname{Erg}(B V(\mathbf{T}), T)) .
$$

Remark 6. We would like to show now that there exists $f \notin B V(\mathbf{T})_{0}$ which is ergodic but is not ergodically stable. To this end we will use the following result proved by Oren in [18] for the cocycles $g_{\beta}=\chi_{[0, \beta)}-\beta, \beta \in[0,1)$ :

If $1, \alpha, \beta$ are linearly independent over the rational numbers, then $T_{g_{\beta}}$ is ergodic.

Choose $\beta$ in such a way that $\left\|q_{n} \beta\right\|$ does not converge to zero. Since $g_{\beta}^{\left(q_{n}\right)}$ takes values $k-q_{n} \beta$ where $k \in \mathbf{Z}$, we have $\left|g_{\beta}^{\left(q_{n}\right)}(x)\right| \geq\left\|q_{n} \beta\right\|$ for every $x$ and so $g_{\beta}^{\left(q_{n}\right)}$ does not converge to zero in measure; hence $f \notin B V(\mathbf{T})_{0}$. On the other hand, for $n \geq 1$ let

$$
g_{n}=g_{\beta}-\frac{1}{n} g_{\{n \beta\}}
$$

Then $g_{n} \rightarrow g$ in $B V(\mathbf{T})$, since $\operatorname{Var}\left(g_{\{n \beta\}}\right)=2$ for all $n$, and $g_{n}$ takes only values in $\frac{1}{n} \mathbf{Z}$, whence $g_{n} \notin \operatorname{Erg}(B V(\mathbf{T}), T)$.

Remark 7. In [14] it is proved that for each irrational rotation by $\alpha$, where $\alpha$ has bounded partial quotients, the set of ergodic absolutely continuous cocycles is a $G_{\delta}$ and dense (in the variation norm) subset of all absolutely continuous cocycles. However in this particular case there is no known example of an absolutely continuous cocycle of type $I I I_{0}$. We leave as a problem if absolutely continuous cocycles of type $I I I_{0}$ do exist in case of bounded partial quotients.

We have been unable to decide whether or not in a pseudo-homogeneous Banach space type $I I I_{0}$ cocycles are always in the closure of coboundaries with measurable transfer functions.

\section{REFERENCES}

[1] J. Aaronson, M. Lemańczyk, Ch. Mauduit, H. Nakada, Koksma's inequality and group extensions of Kronecker transformations, Algorithms, Fractals, and Dynamics, Plenum Press, 1995, 27-50.

[2] J. Aaronson, M. Lemańczyk, D. Volný, A salad of cocycles, preprint. 
[3] I.P. Cornfeld, S.W. Fomin, J.G. Sinai, Ergodic Theory, Springer-Verlag 1982. MR 87f:28019

[4] P. Gabriel, M. Lemańczyk, K. Schmidt, Extensions of cocycles for hyperfinite actions, and applications, to appear in Monatshefte Math.

[5] H. Helson, Cocycles on the circle, J. Operator Th. 16 (1986), 189-199. MR 88f:22020

[6] M. Herman, Sur la conjugaison différentiable des difféomorphismes du cercle à des rotations, Publ. IHES 49 (1979), 5-234. MR 81h:58039

[7] A. Iwanik, M. Lemańczyk, D. Rudolph, Absolutely continuous cocycles over irrational rotations, Isr. J. Math. 83 (1993), 73-95. MR 94i:58108

[8] A.B. Katok, Constructions in Ergodic Theory, unpublished lecture notes.

[9] Y. Katznelson, An Introduction to Harmonic Analysis, Dover, New York 1976. MR 54:10976

[10] A.W. Kočergin, On the homology of functions over dynamical systems, Dokl. AN SSSR 231 (1976). MR 55:3218

[11] L. Kuipers, H. Niederreiter, Uniform Distribution of Sequences, Wiley, 1974. MR 54:7415

[12] J. Kwiatkowski, Factors of ergodic group extensions of rotations, Studia Math. 103 (1992), 123-131. MR 94a:28033

[13] M. Lemańczyk, Ch. Mauduit, Ergodicity of a class of cocycles over irrational rotations, J. London Math. Soc. 49 (1994), 124-132. MR 94m:28028

[14] P. Liardet, D. Volný, Sums of continuous and differentiable functions in dynamical systems, preprint.

[15] M. Lin, B. Sine, Ergodic theory and the functional equation $(I-T) x=y$, J. Operator Theory 10 (1983), 153-166. MR 84m:47015

[16] C. C. Moore, K. Schmidt, Coboundaries and homomorphisms for non-singular actions and a problem of H. Helson, Proc. London Math. Soc. 40 (1980), 443-475. MR 82a:22007

[17] M. G. Nerurkar, On the construction of smooth ergodic skew products, Erg. Th. Dyn. Syst. 8 (1988), 311-326. MR 89m:58123

[18] I. Oren, Ergodicity of cylinder flows arising from irregularities of distribution, Isr. J. Math. 44 (1983), 127-138. MR 84i:10055

[19] D.A. Pask, Skew products over the irrational rotation, Israel J. Math 69 (1990), 65-74. MR 91d:28036

[20] D.A. Pask, Ergodicity of certain cylinder flows, Israel J. Math 76 (1991), 129-152. MR 94a:28034

[21] K. Petersen, Ergodic Theory, Cambridge Univ. Press, 1983. MR 87i:28002

[22] M. Rychlik, The Wiener lemma and cocycles, Proc. Amer. Math. Soc. 104 (1988), 932-933. MR 90a: 28028

[23] K. Schmidt, Cocycles of Ergodic Transformation Groups, Lect. Notes in Math., Vol. 1, Macmillan Co. of India, 1977. MR 58:28262

[24] M. Talagrand, Some functions with a unique invariant mean, Proc. Amer. Math. Soc. 82 (1981), 255-256. MR 83a:28013

[25] W. A. Veech, Strict ergodicity in zero dimensional dynamical systems and the KroneckerWeyl theorem mod 2 , Trans. Amer. Math. Soc. 140 (1969), 1-35. MR 39:1410

[26] D. Volný, Cohomology of Lipschitz and absolutely continuous functions for the circle rotation, preprint.

Department of Mathematics and Computer Science, Nicholas Copernicus University, Ul. Chopina 12/18, 87-100 Toruń, Poland

E-mail address: mlem@mat.uni.torun.pl

Laboratoire d'Analyse, Géométrie et Applications, URA CNRS 742, Université ParisNord, Av. J.-B. Clément, 93430 Villetaneuse, France

E-mail address: parreau@math.univ-paris13.fr

Mathematical Institute, Charles University, Sokolovská 83, 18600 Praha 8, Czech REPUBLIC

E-mail address: dvolny@karlin.mff.cuni.cz 